\title{
A model for multiple outcomes games
}

\author{
Ramzi Ben Larbi Sébastien Konieczny Pierre Marquis \\ CRIL - CNRS, Université d'Artois, France \\ \{benlarbi, konieczny, marquis\}@cril.fr
}

\begin{abstract}
We introduce and study qualitative multiple outcomes games. These games are noncooperative games with qualitative utilities (i.e., values over an ordinal scale), strictly qualitative uncertainty and possible coordination. By strictly qualitative uncertainty, we mean that when there is a set of possible events, the probability of each event is unknown. Coordination is a way offered to the players to remove uncertainty. Qualitative multiple outcomes games is a model for a number of multi-agent problems where agents have minimal information about the interaction effects and where probabilites are unavailable. Among them is multi-agent planning where autonomous planning agents do not share the same goals, and have to generate plans that interact with those of others in a way they cannot unilaterally predict or control.
\end{abstract}

\section{Introduction}

The aim of this work is to introduce and study qualitative outcomes games. In this kind of games, each strategy profile is associated with a set of possible outcomes. For any strategy profile, players are given the possibility to coordinate, that is to agree on a particular element of the associated set of outcomes. Coordination can be achieved only if all the players agree on it. Motivations for introducing coordination in this kind of games are very similar to the ones which motivate the introduction of the notion of correlated equilibrium. The idea is that the players can (cheap) talk about the set of outcomes associated to a strategy profile before choosing their corresponding strategy, so they can coordinate to achieve an interesting outcome for all of them.

When players do not want to (or cannot) agree on an outcome, the outcome that will be realized is any one in the set. One can imagine that an external agent (e.g. Nature) picks up one outcome in the set of possible outcomes. In that case, players do not know the probability distribution used by this agent. Qualitative multiple outcomes games can also prove valuable to model scenarios where it is inappropriate to use a probability distribution on the outcomes. Halpern and Tuttle underline the need for such models without probabilities in [6]. They argue in particular that "some choices in distribued systems must be viewed as inherently nondeterministic (or, perhaps better, nonprobabilistic), and that is inappropriate, both philosophically and pragmatically, to model probabilistically what is inherently nondeterministic". We say that the players' uncertainty about the realized outcome is strictly qualitative. The only available piece of information is the outcome set associated to each strategy profile.

In the following, we make the further assumption that the players' utilities are qualitative. It means that utilities are defined over an ordinal scale, and not necessarily over the set of real numbers. As a consequence, no notion of preference intensity can be derived from the players' utilities.

Let us illustrate the interest for qualitative outcomes games on a modified version of the prisoner's dilemma.

Example 1 Two suspects are arrested by the police. The police has insufficient evidence for a conviction, and offers both prisoners the same deal: if one testifies (strategy D) for the prosecution against the other who remains silent (strategy $C$ ), the betrayer will only have a one year sentence (a satisfaction of 5) and the silent accomplice receives the full 6-year sentence (a satisfaction of 0 )

If both remain silent, the prosecution will be based only on evidences, so the result of the trial will depend of the convincingness of the suspects. If they are not sufficiently convincing, they will go only 3 years in jail (a satisfaction of 3 ), but if they give coherent stories (by the means of any coordination process), they will go free (a satisfaction of 6).

If each betrays the other, each one will receive a 4 or 5 year sentence (a satisfaction of 2 or 1 respectively), depending of the suspects testimony during the trial. Thus, again, they can agree on a story which does not prevent them from going to jail but ensures them the less possible sentence.

In this scenario, players are confronted with situations leading to different outcomes. Players can coordinate on a particular outcome. If they do not, any possible outcome may be realized.

In strategic form, the associated game can be represented as in Table 1. In this game, there is some uncertainty on the outcome obtained with the profile of strategies $(C, C)$, and the one obtained with the profile $(D, D)$. This uncertainty cannot be probabilized, and can be removed if the two agents 


\begin{tabular}{|c|c|c|}
\hline & $\mathrm{C}$ & $\mathrm{D}$ \\
\hline $\mathrm{C}$ & $(6,6)$ & $(0,5)$ \\
& $(3,3)$ & \\
\hline $\mathrm{D}$ & $(5,0)$ & $(1,1)(1,2)$ \\
& & $(2,1)(2,2)$ \\
\hline
\end{tabular}

\section{Table 1. Prisoner's dilemma with uncertainty and possible coordination}

coordinate. Note that for most probability distributions on outcomes sets, this game is a prisoner's dilemma.

As we will see, coordination modifies the usuual picture since, thanks to it, the profile $(C, C)$, and the outcome $(6,6)$ are achievable.

These qualitative games can model a number of situations from multi-agent planning problems where there is no central planner and agents are autonomous, meaning that each one has her own goals and is willing to coordinate only if it is of her interest (in this respect these problems differ from task sharing ones).

In such problems each agent (player) has a set of possible actions (moves), and goals (represented for instance by an utility function). She can generate plans (sequences of actions) in order to reach the best possible consequence from her point of view. When only one agent is considered, the problem of finding the best plan is a classical planning problem, well-studied in artificial intelligence. But when several agents must be considered, the problem of finding the best plan for each agent is much more complex since plans may interact, and requires tools from game theory. When agents have to compute their plans before knowing the other players' choice, cannot change their plans after it, do not know the exact execution order of the actions of the whole profile of plans (except for their own actions), and when several players may agree on building mutual plans (removing the uncertainty at the execution level, exactly as if they would consist of one - global - agent), the planning problem can be modeled using our qualitative games. For more details on such multi-agent planning problems see [4].

The following example depicts such a situation.

Example 2 Two agents, a robot-painter and an robotelectrician, operate in a single room. The bulb has to be changed (which is the goal of the electrician) and the ceiling has to be painted (which is the goal of the painter). The electrician has a new bulb and the painter the materials needed to paint the ceiling. Now, there is a single ladder in the room (the ladder is thus a critical resource). Furthermore, the painter needs some light in the room in order to make her job. The electrician can achieve three actions: TLe ("take the ladder"), CB ("change the bulb"), RLe ("release the ladder"); and the painter three actions: TLp ("take the ladder"), P ("paint”), RLp ("release the ladder") ; P succeeds only if CB has been performed before. TLe and TLp succeed only if the ladder is available (i.e., it has been released before).

The following interactions can be easily envisioned:

- If the painter takes the ladder first, then she will not be able to achieve her goal (since the bulb has to be changed first); if she does not release the ladder, then the electrician will not be able to achieve her goal.

- If the electrician takes the ladder first, she will be able to achieve her goal; then, the painter will be able to achieve her goal if and only if the electrician releases the ladder. Accordingly, if both agents coordinate so as to execute the joint plan TLe.CB.RLe.TLp.P, then both agents will be satisfied.

If the robot-painter puts forward the plan $p=$ TLp.P.RLp, she is only ensured that the actions of $p$ will be executed in the desired order. While she knows the electrician goal and actions, she does not know which plan the electrician will choose. Indeed, the set of feasible plans for an agent is not a singleton in general. Even if this set is a singleton, the painter still ignores the execution ordering, i.e., how her plan will interact with the electrician's one. The resulting uncertainty dissapears whenever the two agents coordinate to put forward a common plan.

The contribution of the present paper is twofold: we introduce qualitative multiple outcomes games with coordination, and show how to solve them.

The rest of the paper is as follows. In Section 2 we give some preliminaries. Then we define qualitative multiple outcomes games in Section 3. In Section 4 and 5 we explain how to solve such games. We illustrate the resolution process on examples in Sections 6 and 7. We discuss the discriminating power of our model in Section 8 by studying the size of the evaluation scale. We also discuss connections between qualitative multiple outcomes games and standard games in Section 9.

\section{Preliminaries}

Let $\leq$ be a preorder on a set $\chi$, i.e., $\leq$ is a reflexive and transitive relation. The associated strict order relation $<$ and the equivalence relation $\sim$ are defined respectively by:

$\mathrm{x} \sim \mathrm{y}$ iff $\mathrm{x} \leq \mathrm{y}$ and $\mathrm{y} \leq \mathrm{x}$; and $\mathrm{x}<\mathrm{y}$ iff $\mathrm{x} \leq \mathrm{y}$ and $\mathrm{y} \not \leq \mathrm{x}$.

Given $\mathrm{n}$ relations $\leq_{i}, i \in[1, n]$ on $\chi$, the associated lexicographic relation $\operatorname{lex}\left(\leq_{1}, \cdots, \leq_{n}\right)$ is defined by

$\mathrm{x} \leq_{\text {lex }\left(\leq_{1}, \cdots, \leq_{n}\right)}$ y iff $\mathrm{x}<_{\text {lex }\left(\leq_{1}, \cdots, \leq_{n}\right)}$ y or

$\mathrm{X} \sim_{\text {lex }\left(\leq_{1}, \cdots, \leq_{n}\right)} \mathrm{y}$ with:

- $\mathrm{x}<_{l e x\left(\leq_{1}, \cdots, \leq_{n}\right)}$ y iff $\exists j \in[1, n]$ s.t. $\forall i<j \mathrm{x} \sim_{i} \mathrm{y}$ and $\mathrm{x}<j \mathrm{y}$.

- $\mathrm{x} \sim_{l e x\left(\leq_{1}, \cdots, \leq_{n}\right)}$ y iff $\forall i \in[1, n] \mathrm{x} \sim_{i} \mathrm{y}$. 
We denote by $\subseteq$ the inclusion relation between sets and $C$ is used for strict inclusion. For a given set $\chi$, we denote $2^{\chi}$ the power set of $\chi$.

Let $x=\left\langle x_{1}, \cdots, x_{n}\right\rangle$ and $y=\left\langle y_{1}, \cdots, y_{n}\right\rangle$ be two vectors in $\chi^{n}$. y Pareto dominates $x$ iff $\forall i \in[1, n], x_{i} \leq$ $y_{i}$ and $\exists i \in[1, n], x_{i}<y_{i}$. The Pareto front of a set $E \subseteq \chi^{n}$ is the subset of $E$ formed by its non-Pareto dominated points, noted $\mathrm{pf}(E)$, i.e. $\mathrm{pf}(E)=\{x \in E \mid \nexists y \in$ $E y$ Pareto dominates $x\}$.

We denote $\min (\chi, \leq)$ the set of minima of $\chi$ and $\max (\chi$, $\leq)$ the set of maxima of $\chi$ with respect to the relation $\leq$, i.e., $x \in \min (\chi, \leq)$ iff $\nexists y \in \chi$ s.t. $y<x$, and $x \in \max (\chi, \leq)$ iff $\nexists y \in \chi$ s.t. $x<y$.

Let $x=\left\langle x_{1}, \cdots, x_{n}\right\rangle$ and $y=\left\langle y_{1}, \cdots, y_{n}\right\rangle$ be two vectors in $\chi^{n}$, and let $\leq$ be a relation on $\chi$, then $x \leq^{i}$ $y$ is a notation for $x_{i} \leq y_{i}$, and $x_{-i}$ is a notation for $\left\langle x_{1}, \cdots, x_{i-1}, x_{i+1}, \cdots, x_{n}\right\rangle$.

\section{Qualitative Multiple Outcomes Games}

Let us now define the notion of qualitative multiple outcomes game on which we focus. We start from the usual notion of strategic form games.

Definition 1 (Strategic form game) $A$ game in strategic form is a 3-tuple $\Gamma=\left(N,\left(S_{i}\right)_{i \in[1, n]}, \mu\right) . \quad N$ is a set of players, $S_{i}$ is a non-empty set of strategies of a player $i$, $S=\times_{i \in N} S_{i}$ is the set of strategy profiles and $\mu: S \longrightarrow \mathbb{R}^{n}$ is the game utility function which associates $s \in S$ to an outcome $\mu(s)=\left(\mu_{1}(s), \cdots, \mu_{n}(s)\right)$. Hence, $\mu_{i}(s)$ is player $i$ utility for the profile $s \in S$.

In order to go from standard strategic form games to multiple outcomes games, the idea is to associate each profile to a non-empty finite set of outcomes:

Definition 2 (Multiple outcomes game) A multiple outcomes game in strategic form is a 3-tuple $\Gamma=$ $\left(N,\left(S_{i}\right)_{i \in[1, n]}, U\right) . \quad N$ is a set of players, $S_{i}$ is a nonempty set of strategies of a player $i, S=\times_{i \in N} S_{i}$ is the set of strategy profiles and $U: S \longrightarrow 2^{\mathbb{R}^{n}} \backslash\{\emptyset\}$ is the game utility function which associates $s \in S$ to a finite outcomes set.

Since we are concerned with qualitative games, we generalize the previous definition to any qualitative utility (i.e., utilities do not necessarily belong to the set of real numbers).

\section{Definition 3 (Qualitative multiple outcomes game)}

Let $\chi_{1}, \cdots, \chi_{n}$ be sets totally ordered by, respectively $\leq_{1}, \cdots, \leq_{n}$. A multiple outcomes game in strategic form is a 3-tuple $\Gamma=\left(N,\left(S_{i}\right)_{i \in[1, n]}, U\right) . N$ is a set of players, $S_{i}$ is a non empty set of strategies of a player $i, S=\times_{i \in N} S_{i}$ is the set of strategy profiles and $U: S \longrightarrow 2^{\chi_{1} \times \cdots \times \chi_{n}} \backslash\{\emptyset\}$ is the game utility function which associates $s \in S$ to a finite outcomes set.
Note that the "utility scale" $\leq_{i}$ of player $i$ can be different from the scales used by other players. For the ease of reading, abusing words, we will call numbers the elements of the sets $\chi_{i}$.

\section{Solving Qualitative Multiple Outcomes Games}

To solve qualitative multiple outcomes games, the important point is to know how a player evaluates a given strategy profile. When a player compares different profiles, there are several dimensions to take into account because of their impact on her evaluation. These dimensions are:

- The player utility for this profile: Remind that, in multiple outcomes games, a profile does not usually give a unique outcome, but a set of outcomes. Thus the player utility for a given profile is not a unique number, but a set of such numbers, one for each possible outcome. We call this set the player projection of the profile. In order to compare different profiles, one thus has to be able to compare such projections (sets of numbers). There is no unique, canonical way to do it. This will lead to several possible definitions.

- The possibility of a coordination: We assume that the players can remove the uncertainty associated to a profile, if they all agree to do so (by coordinating). As the players are supposed to be rational ones, coordination will occur only when it benefits to all players ${ }^{1}$. So, in case of coordination, it does not make sense to consider the whole projection set of a player, only the possible coordination outcomes (what we call consensus points) are important.

- The other players outcome uncertainty: Suppose that, in a two-player game, we have the three following profiles

-a- $(0,0)(1,1)$

-b- $(0,1)(1,1)$

-c- $(0,1)(1,0)$

that are represented graphically on Figure 1.

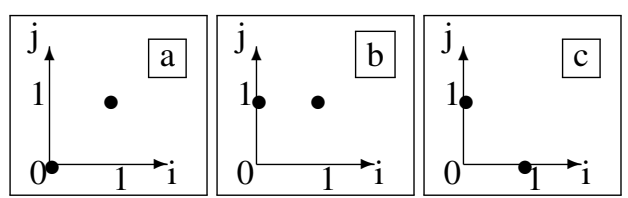

Figure 1. Possible set of outcomes

For these three profiles the projection of player 1 is the same $(\{0,1\})$, but the projection of player 2 is different. The point is to determine whether player 1 has to evaluate differently these three situations. Clearly profile $c$ is the less

\footnotetext{
${ }^{1}$ More exactly coordination is not irrational when it benefits to some players, and when the others are indifferent to it.
} 
interesting one for player 1 since there will be no possibility of coordination using this profile. Indeed, in profile $c$ the two players are clearly antagonistic (one player gets 0 when the other gets 1 ), and there will be no hope in finding a coordination. Now the point is to determine whether there is a difference between profiles $a$ and $b$ for player 1 . In both profiles, the agents can coordinate to reach the outcome $(1,1)$. But, when in profile $a$ it is of the two players interest to reach the $(1,1)$ outcome, in profile $b$ player 2 has no reason to reject a coordination. Still 2 has no strong incentive to accept it neither. So profile $a$ is better for player 1 than the profile $b$ since player 2 will have more incentive to coordinate. Thus, in order to discriminate these two profiles, player 1 has to take into account player 2 outcome uncertainty when she evaluates the profiles.

We claim that these three dimensions are the only ones which matter in a player evaluation of a profile. We now show how to model these dimensions, and how to take them into account in order to solve these qualitative multiple outcomes games.

Let us first define formally the notion of projection.

Definition 4 (Projection) Let $\Gamma=\left(N,\left(S_{i}\right)_{i \in[1, n]}, U\right)$ be a qualitative multiple outcomes game. Let $s \in S$ be a strategy profile. The projection with respect to $i \in[1, n]$ of $U$ gives the possible utilities of player $i$ for each profile $s \in S$. It is a mapping $U_{i}: S \longrightarrow 2^{\chi_{i}} \backslash\{\emptyset\}$.

We need to identify the possibilities of coordination. This is the aim of consensus points.

Definition 5 (Consensus points) Let $\Gamma=\left(N,\left(S_{i}\right)_{i \in[1, n]}\right.$, $U)$ be a qualitative multiple outcomes game. Let $s \in S$ be a strategy profile. A consensus point for $s$ is an outcome $w \in U(s)$ s.t. $\exists J \subset N, \forall j \in J, \forall w^{\prime} \in U(s), w_{j}^{\prime} \sim w_{j}$ and $\forall i \in N \backslash J, \exists w " \in U(s)$ s.t. $w_{i}^{\prime \prime}<w_{i}$. The set of all consensus points associated to $s$ is denoted by $\operatorname{cp}(s)$.

Consensus points are simply outcomes such that for each player, either there is at least a worse outcome, or all the outcomes of the profile are equivalent. Thus, when a consensus point exists, all players have an interest for coordination (since it guarantees to avoid the worst outcome), or at least no interest to refuse it (all outcomes are equivalent). The consensus points are strongly related to the strict individually rational points commonly considered in bargaining theory (see e.g. [10]). In this theory strict individual rationality SIR requires that a player considers only these points in the bargaining set that she strictly prefers to what she can expect if there is no agreement. This is a basic assumption one can make about players if willing to define a rational solution. SIR can be translated into our framework through the following requirement. A player is willing to coordinate on an outcome $w$ belonging to some strategy profile $s$ associated outcomes set $U(s)$ only if its expected reward $w_{i}$ is strictly better than the minimum of her projection of the outcome set, which is the worst reward she may obtain without coordination. The second part of the above definition specifies that a player $i$ may coordinate on an outcome when it strictly dominates another element of the outcomes set with respect to her preference relation $\leq_{i}$. That obviously meets the SIR requirement.

The first part of the definition of consensus points is about the case where all the outcomes in the outcomes set are of equal value for a player. In this case, the player has no reason to refuse coordination since there is no other outcome that she prefers (as usual, we suppose that all the preferential information is encoded by the utility function).

The following proposition makes explicit the relation between the consensus points of a strategy profile and the Pareto-optimal points:

Proposition 1 Let $\Gamma=\left(N,\left(S_{i}\right)_{i \in[1, n]}, U\right)$ be a qualitative multiple outcomes game. Let $s \in S$ be a strategy profile.

$$
\operatorname{pf}(\mathrm{cp}(s)) \subseteq \operatorname{pf}(s) .
$$

We also have that:

Proposition 2 Let $\Gamma=\left(N,\left(S_{i}\right)_{i \in[1, n]}, U\right)$ be a qualitative multiple outcomes game. Let $s \in S$ be a strategy profile. If $\operatorname{cp}(s) \neq \emptyset$ then

$$
\operatorname{cp}(s) \cap \operatorname{pf}(s) \neq \emptyset .
$$

Thus among consensus points are some Pareto-optimal points, but not all of them in general; it is also not the case in general that every consensus point is Pareto-optimal.

As explained above, when a coordination is possible, the full player projection of the profile is not interesting. The set $\gamma(s)$ of relevant outcomes of a strategy $s$ is defined as the set of its Pareto-optimal consensus points, when this set is not empty:

Definition 6 (Relevant outcomes) Let $\Gamma=\left(N,\left(S_{i}\right)_{i \in[1, n]}\right.$, $U)$ be a qualitative multiple outcomes game. Let $s \in S$ be a strategy profile.

$$
\gamma(s)= \begin{cases}U(s) & \text { if } \mathrm{cp}(s)=\emptyset \\ \operatorname{pf}(\operatorname{cp}(s)) & \text { otherwise }\end{cases}
$$

The reason to select only Pareto-optimal consensus points is obvious: if the players agree for a coordination, then they will choose an outcome among the the most interesting ones. One can note that we state that players will not simply agree on any Pareto-optimal point of the outcome set. This is because one outcome can be Pareto-optimal and still unlikely to be the result of a coordination. In Figure 2 Pareto optimal points are $a, b, c$. It is obvious that player $i$ will never agree on point $c$ since its projection for $i$ is minimal. So will do player $j$ with point $b$. So the only relevant outcome in this case is point $a$.

Now, suppose that we have a preorder $\leq_{c}$ for comparing sets of numbers (we will give some examples of such relations in the next section). We can now use it to evaluate every 


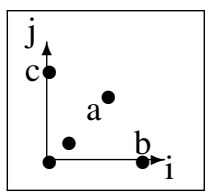

Figure 2. Relevant outcomes

profile. More exactly, as the evaluation is qualitative, we can evaluate each pair of profiles, by constructing a relation $\preccurlyeq_{i}$, that indicates how to compare profiles for player $i$.

$\leq_{c}$ allows to compare the player utilities for the two profiles under consideration. In order to take into account possible coordination and other players outcome uncertainty, $\leq_{c}$ has to be refined in two ways:

- The first one amounts to comparing different coordination possibilities:

Definition 7 (Relevant outcomes criterion) Let $_{c_{i}}$ be $a$ relation over sets of numbers. Then the associated relation $\leq_{\gamma_{c_{i}}}$ is defined by: for any strategy profiles $s_{1}$ and $s_{2}$,

$$
s_{1} \leq_{\gamma_{c_{i}}} s_{2} \text { iff } \gamma\left(s_{1}\right) \leq_{c_{i}} \gamma\left(s_{2}\right) .
$$

- The second one evaluates the number of other players which have no outcome uncertainty for a given profile:

Definition 8 (Dependence criterion) Let $\Gamma=(N$, $\left.\left(S_{i}\right)_{i \in[1, n]}, U\right)$ be a qualitative multiple outcomes game. Let $i$ be a player, $s$ a strategy profile and $E(s, i)=$ $\left\{j \in N \backslash\{i\}\right.$ s.t. $\forall w, w^{\prime} \in U(s), w \sim_{j} w^{\prime}$ and $\exists w, w^{\prime} \in$ $\left.U(s), w<_{i} w^{\prime}\right\}$. The relation $\leq_{d(i)}$ over the strategy profiles is defined by: let $s_{1}$ and $s_{2}$ be two strategy profiles,

$$
s_{1} \leq_{d(i)} s_{2} \text { iff }\left\{\begin{array}{l}
\left|E\left(s_{1}, i\right)\right| \geq\left|E\left(s_{2}, i\right)\right| \\
o r \\
\operatorname{cp}\left(s_{1}\right)=\emptyset
\end{array}\right.
$$

This refinement is only useful when there are consensus points (this explains the second condition of the definition); it discriminates the cases where there is a mutual interest for coordination between all players, and the cases where some players are indifferent (so they have no a priori reason to reject coordination, but they have no strong incentive to accept it). Clearly, the less indifferent players, the better.

On this ground, we are ready to define the final player evaluation for the profiles:

Definition 9 (Player evaluation) Let $\Gamma=\left(N,\left(S_{i}\right)_{i \in[1, n]}\right.$, $U)$ be a qualitative multiple outcomes game. Let $i \in N$ and $\leq_{c_{i}}$ a preorder on sets of numbers. The evaluation by player $i$ of the strategy profiles of $S$ is given by the relation $\preccurlyeq_{i}^{c}=\operatorname{lex}\left(\leq_{\gamma_{c_{i}}}, \leq_{c_{i}}, \leq_{d(i)}\right)$.

The use of $\operatorname{lex}\left(\leq_{\gamma_{c_{i}}}, \leq_{c_{i}}, \leq_{d(i)}\right)$ allows us to adequately take into account the three dimensions of the evaluation. We first start by comparing the set of numbers corresponding to each projection, but by focusing only on points of interest when a coordination is possible in the profile. For profiles that are equivalent for this first criterion, we look at the whole projection. And when these two profiles are still equivalent, we look at cases where other agents are indifferent in one profile, which are less interesting than when they all really need coordination.

With this evaluation one can now use all notions of solutions from classical game theory, such as Nash equilibria, in order to solve these games.

When the evaluation relation $\preccurlyeq_{i}^{c}$ is a total one, one can represent it, and its corresponding ranking, using real numbers, and just solve the corresponding classical strategic form game. Of course mixed strategies cannot be used, since preference intensities cannot be infered here (this contrasts with usual utilities).

\section{Proposition 3}

- If $\leq_{c_{i}}$ is a preorder over sets of numbers, then $\preccurlyeq_{i}^{c}$ is a preorder over strategy profiles.

- If $\leq_{c_{i}}$ is total then $\preccurlyeq_{i}^{c}$ is total.

Note that, even if the evaluation relation is partial, one can still use game theory notions, such as Nash equilibrium, to solve these games. This can be expressed as follows for partial orders:

Definition 10 (Nash equilibrium) Let $\Gamma=\left(N,\left(S_{i}\right)_{i \in[1, n]}\right.$, $U)$ be a qualitative multiple outcomes game. A profile of strategies $s$ is a Nash equilibirum if no agent can get a better evaluation by choosing another strategy, i.e., $s$ is a Nash equilibrium if and only if $\nexists i \in N \nexists s^{\prime} \in S_{i}$ s.t $\left(s_{-i}, s_{i}\right) \prec_{i}$ $\left(s_{-i}, s^{\prime}\right)$.

\section{Comparing two sets of numbers}

In this section we address the problem of comparing two sets of numbers. There are several different ways to define this comparison (cf. e.g. [3]).

A first possibility is a worst-case comparison of the sets, which is usual in strict uncertainty cases.

Definition 11 (Min criterion) Let $\Gamma=\left(N,\left(S_{i}\right)_{i \in[1, n]}, U\right)$ be a qualitative multiple outcomes game. Let $s_{1}$ and $s_{2}$ be two strategy profiles. For $i \in[1, n]$,

$$
s_{1} \leq_{\min _{i}} s_{2} \text { iff } \min \left(U_{i}\left(s_{1}\right), \leq_{i}\right) \leq_{i} \min \left(U_{i}\left(s_{2}\right), \leq_{i}\right) .
$$

This min criterion is in a sense the safest one in case of complete ignorance. It is usually known as Wald criterion [11].

This criterion is refined by the minmax one, obtained by lexicographically refining the min thanks to the maximal possible outcomes. 
Definition 12 Let $\Gamma=\left(N,\left(S_{i}\right)_{i \in[1, n]}, U\right)$ be a qualitative multiple outcomes game. Let $s_{1}$ and $s_{2}$ be two strategy profiles. For $i \in[1, n]$,

$$
s_{1} \leq_{\max _{i}} s_{2} \text { iff } \max \left(U_{i}\left(s_{1}\right), \leq_{i}\right) \leq_{i} \max \left(U_{i}\left(s_{2}\right), \leq_{i}\right) .
$$

Definition 13 (Minmax criterion) Let $\Gamma=\left(N,\left(S_{i}\right)_{i \in[1, n]}\right.$, $U)$ be a qualitative multiple outcomes game. Let $s_{1}$ and $s_{2}$ be two strategy profiles.

$$
s_{1} \leq_{\text {minmax }_{i}} s_{2} \text { iff } s_{1} \leq_{l e x\left(\leq_{\min _{i}}, \leq_{\max _{i}}\right)} s_{2} .
$$

The above two criteria define total preorders, so they allow to fully rank all the profiles.

The next one is a criterion based on interval relations [5]. Its main drawback is that the corresponding preorder is only a partial relation.

Definition 14 (Product criterion) Let $\Gamma=\left(N,\left(S_{i}\right)_{i \in[1, n]}\right.$, $U)$ be a qualitative multiple outcomes game. Let $s_{1}$ and $s_{2}$ be two strategy profiles. For $i \in[1, n]$,

$$
s_{1} \leq_{\text {product }_{i}} s_{2} \text { iff } s_{1} \leq_{\min _{i}} s_{2} \text { and } s_{1} \leq_{\max _{i}} s_{2} .
$$

\section{Solving the prisoner's dilemma example}

Let us now explain how to model and solve Example 1 using our framework.

Let us use the min criterion. For player 1 we have the following projections of the strategy profiles:

$U_{1}((C, C))=\{3,6\}, U_{1}((C, D))=\{0\}$,

$U_{1}((D, C))=\{5\}, U_{1}((C, C))=\{1,2\}$.

We have the following sets of relevant outcomes for the strategy profiles:

$\gamma((C, C))=\{(6,6)\}, \gamma((C, D))=\{(0,5)\}$,

$\gamma((D, C))=\{(5,0)\}, \gamma((C, C))=\{(2,2)\}$.

The first comparison of strategy profiles (using $\leq_{\gamma_{c_{i}}}$ ) gives here directly a strict order on profiles. So the lexicographic relation $\preccurlyeq_{1}$ is such that

$$
(C, D) \prec_{1}(D, D) \prec_{1}(D, C) \prec_{1}(C, C) .
$$

The two players being symmetric, we deduce the following order on strategy profiles for player 2 :

$$
(D, C) \prec_{2}(D, D) \prec_{2}(C, D) \prec_{2}(C, C) .
$$

It is easy to check that the profile $(C, C)$ is the only Pareto dominant Nash equilibrium of this game. So the solution for this qualitative multiple outcomes game is that the players choose strategy $C$ and coordinate (on a consistent story) which will allow them to go free.

As the min criterion gives here a strict order on strategy profiles, using any other criterion that refines it (such as minmax and product criteria) leads to the same solution.
When the relations $\preccurlyeq_{i}$ are total ones, it is possible to translate qualitative multiple outcomes game into classical strategic form games, simply by assigning ranks to the equivalence classes. Thus we obtain the game in strategic form of Table 2 which is no longer a prisoner's dilemma. Our procedure allows us to envision the situation as a coordination game. One can easily check that $(C, C)$ is a Nash equilibrium.

\begin{tabular}{|c|c|c|}
\hline & $\mathrm{C}$ & $\mathrm{D}$ \\
\hline $\mathrm{C}$ & $(4,4)$ & $(1,3)$ \\
\hline $\mathrm{D}$ & $(3,1)$ & $(2,2)$ \\
\hline
\end{tabular}

\section{Table 2. The corresponding strategic form game}

\section{A more complex example}

In this section we present a qualitative multiple outcomes game where the full power of the lexicographic nature of the evaluation function as defined earlier is at work. This example allows us to illustrate how the three relations forming the player evaluation work together.

Example 3 Let us use the min criterion to solve the qualitative multiple outcomes game given in Table 3.

\begin{tabular}{|c|c|c|}
\hline & C & D \\
\hline A & $(1,3)(1,1)$ & $(1,2)(3,2)$ \\
& $(3,3)$ & \\
\hline B & $(0,3)(0,1)$ & $(1,1)$ \\
& $(3,3)$ & \\
\hline
\end{tabular}

\section{Table 3. A more complex example}

For player 1, the first comparison of strategy profiles (using $\left.\leq_{\gamma_{\text {min }}}\right)$ gives here :

$(B, D)<_{\gamma_{\text {min }}}(A, C) \sim_{\gamma_{\text {min }}}(A, D) \sim_{\gamma_{\text {min }}}(B, C)$.

We then use the second criterion (using $\leq_{m_{i n}}$ ) which gives: $(B, C)<_{\text {min }_{1}}(A, C) \sim_{\text {min }_{1}}(A, D)$.

Finally the third criterion (using $\left.\leq_{d(1)}\right)$ gives:

$(A, C)<_{\operatorname{dep}(1)}(A, D)$

(since $|E((A, C), 1)|=0$ and $|E((A, D), 1)|=1)$.

So the lexicographic relation $\preccurlyeq_{1}$ is such that $(B, D) \prec_{1}(B, C) \prec_{1}(A, D) \prec_{1}(A, C)$.

For player 2 , the first comparison of strategy profiles (using $\left.\leq_{\gamma_{\text {min }_{2}}}\right)$ gives here :

$(B, D)<_{\gamma_{\text {min }}}(A, D)<_{\gamma_{\text {min }_{2}}}(A, C) \sim_{\gamma_{\min _{2}}}(B, C)$.

The second criterion (using $\leq$ min $_{2}$ ) gives:

$(A, C) \sim_{\text {min }_{2}}(B, C)$.

Finally the third comparison (using $\left.\leq_{d(2)}\right)$ gives:

$(A, C) \sim_{d(2)}(B, C)$. 
So the lexicographic relation $\preccurlyeq_{2}$ is such that

$(B, D) \prec_{2}(A, D) \prec_{2}(A, C) \sim_{2}(B, C)$.

It is easy to check that the profile $(A, C)$ is the only Nash equilibrium of this game. So the solution of this game is that player 1 chooses strategy $A$, player 2 chooses $C$, and they coordinate on the outcome $(3,3)$.

\section{Size of the evaluation scale}

As often with solution concepts, there is no formal proof that our model for solutions is the right one. Instead, let us give some arguments in favour of it. In this section, we focus on the number of equivalence classes induced by $\preccurlyeq_{i}^{c}$ and we show it is big enough. Because even if the solution concept is rational, if the preorder divides the set of strategy profiles into a very small number of subsets it will be of little help to a player who wants to make a decision based on it. In the following section, we show that our notion of solution is strongly correlated to other notions of solutions commonly accepted in game theory.

Let us begin by considering a player evaluation of the possible profiles (with multiple outcomes), when the utilities of the agents are binary ones, i.e., 0 (unsatisfied), and 1 (satisfied). For such games, with 2 players, there are 4 outcomes $(0,0),(0,1),(1,0),(1,1)$, and 15 different outcomes sets. They are represented on Figure 3.

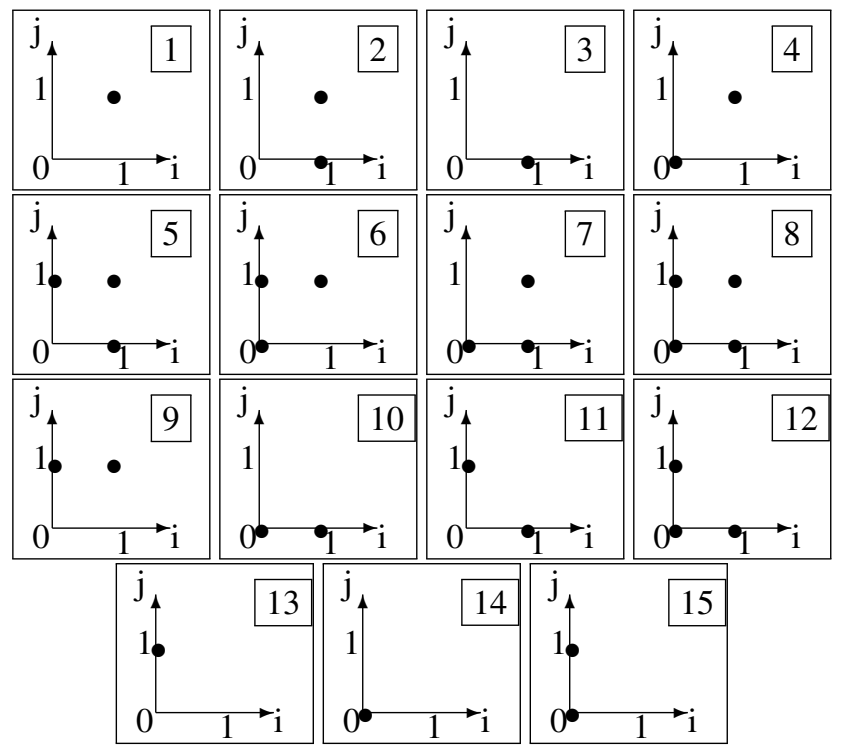

Figure 3. All possible outcomes sets in the twoplayer binary case

With the min criterion, the player evaluation ranks the 15 outcome sets in 4 different equivalence classes. With the minmax criterion, the player evaluation ranks the 15 outcome sets in 5 different equivalence classes. The following straightforward propostion states that minmax and the product criteria are always more discriminating than the min criterion.

Proposition 4 Let $\Gamma=\left(N,\left(S_{i}\right)_{i \in[1, n]}, U\right)$ be a qualitative multiple outcomes game. Let $i \in N$ and $s, s^{\prime} \in S$. Then $s \preccurlyeq_{\text {minmax }_{i}} s^{\prime} \Rightarrow s \preccurlyeq_{\text {min }_{i}} s^{\prime}$ and $s \preccurlyeq_{\text {product }_{i}} s^{\prime} \Rightarrow s \preccurlyeq_{\text {min }_{i}} s^{\prime}$.

We can easily compute the numbers of different equivalence classes for the player evaluation when the min criterion is used and the scales are finite:

Proposition 5 If the evaluation scale of all players is of cardinality $m$, then using the min criterion the equivalence relation induced by $\preccurlyeq_{\text {min }_{i}}$ has $m^{2}$ equivalence classes in the 2-player case, and more generally, it has $\frac{n}{2} * m^{2}+\left(1-\frac{n}{2}\right) * m$ different equivalence classes in the n-player case.

From proposition 4 it comes that with the minmax and product criteria the number of equivalence classes cannot get lower.

\section{Links with classical game theory}

In this section we give some results which illustrate the relationship between qualitative multiple outcomes games and usual strategic form games. For the sake of comparison we suppose here that utilities are real numbers.

The first proposition is a straightforward one, stating that qualitative multiple outcomes games are a generalisation of (qualitative) strategic form games.

Proposition 6 When in a qualitative multiple outcomes games $\Gamma$, there is only one outcome associated to each strategy profile, the (pure) Nash equilibria of $\Gamma$ viewed as a qualitative multiple outcomes game are exactly the (pure) Nash equilibria of $\Gamma$ viewed as a classical game.

In qualitative multiple outcomes games, the uncertainty on the exact outcome can be modeled by adding an additional player, say Nature, which chooses the exact outcome for each profile with a given probability, unknown by the players, when they do not coordinate. Let us call this representation of the problem the associated extended game, and let us explore some results about this representation.

Proposition 7 Let $\Gamma$ be a qualitative multiple outcomes game. If a strategy profile is a Nash equilibrium of the associated extended game for any probability distribution on the outcomes then this strategy profile is a Nash equilibrium of $\Gamma$.

This result means that if a strategy profile is "objectively" (i.e., whatever the realized outcome) a Nash equilibrium then it is recognized as such in the setting of qualitative multiple outcomes game.

There is also a dual result, stating some rationality of the Nash equilibria of the qualitative multiple outcomes games. 
Proposition 8 If a strategy profile is a Nash equilibrium of a qualitative multiple outcomes game, then there exists a probability distribution on the outcomes such that this strategy profile is a Nash equilibrium of the corresponding associated extended game.

So if a profile is not a Nash equilibrium of any associated extended game then it is not a Nash equilibrium of the qualitative outcomes game.

\section{Conclusion}

In this paper we have introduced qualitative multiple outcomes games. In such games players' utilities are qualitative ones, there is a strict uncertainty on the outcome of each profile of strategies, and this uncertainty can be removed if the agents agree to coordinate.

Solving these games requires to evaluate the strategy profiles for each player. Such an evaluation needs to take three different pieces of information into account: the utility of the player, the possibilities of coordination, and the utility of the other players.

Qualitative multiple outcomes games can be used to model scenarios where an autonomous agent has to build a plan while taking account for the uncertainty introduced by the possible interaction with other agents' plans. In this setting, the solution of the game should be an optimal plan for the agent. Due to the lack of space, we cannot elaborate more on this point here. But we refer the reader to [4] for the case where agents have dichotomous preferences.

Several works addressed the problem of decision making under strict uncertainty (cf. e.g. $[1,9,8]$ ). But it seems that there are no such works in game theory. It should be interesting to see how these approaches apply in our qualitative games framework. In particular, the different evaluations we propose in this paper all satisfy Arrow-Hurwicz conditions [1] (if we consider only one player). So Arrow-Hurwicz strict uncertainty decision framework can be seen as the underlying decision theory behind qualitative multiple outcomes games, just like Von Neumann and Morgenstern expected utility is for classical game theory.

An underlying hypothesis of this work is that there is no coordination cost, since if a player is indifferent between all the outcomes, we suppose that she does not refuse coordination. Things would change if this assumption was removed and a (even small) cost for coordination must be considered. In this case an agent which is indifferent between all the outcomes should refuse coordination if she is rational. Refining our framework for taking account for such coordination costs is a perspective for further research.

Another direction for future work is to study the links between the setting of qualitative multiple outcomes games and that of games with strict type uncertainty used in [2] and [7].

Finally, we have so far modelled situations where players have strict qualitative uncertainty on the outcomes sets. We think that qualitative multiple outcomes games can be used to study the more general case of partial knowledge by taking inspiration from hurwicz proposal in the decision making problem to model partial knowledge by eliminating some probability distributions on the possible states of the world from the set of all possible ones (c.f. [8] for a discussion). The resulting subset (which contains "plausible" probability distributions) can then be considered as a set of new states on which the agent has a strict uncertainty. We plan to adapt this proposal to our setting where the probabilities are on outcomes sets.

\section{References}

[1] K. J. Arrow and L. Hurwicz. An optimality criterion for decision-making under ignorance. In Studies in Resource Allocation Processes, pages 463-471. Cambridge University Press, 1977.

[2] I. Ashlagi, D. Monderer, and M. Tennenholtz. Resource selection games with unknown number of players. In International Conference on Autonomous Agents and Multiagent Systems (AAMAS'06), pages 819-825, 2006.

[3] S. Barbera, W. Bossert, and P. K. Pattanik. Ranking sets of objects. Technical report, Universite de Montreal, Departement de sciences economiques, 2001. available at http://ideas.repec.org/p/mtl/montde/2001-02.html.

[4] R. Ben Larbi, S. Konieczny, and P. Marquis. Extending classical planning to the multi-agent case: A gametheoretic approach. In European Conference on Symbolic and Quantitative Approaches to Reasoning with Uncertainty (ECSQARU'07), pages 731-742, 2007.

[5] P. Fishburn. Interval Orders and Interval Graphs. J. Wiley, New York, 1985.

[6] J. Halpern and M. Tuttle. Knowledge, probability, and adversaries. J. ACM, 40(4):917-962, 1993.

[7] N. Hyafil and C. Boutilier. Regret minimizing equilibria and mechanisms for games with strict type uncertainty. In International Conference on Uncertainty in Artificial Intelligence (UAI'04), pages 268-277, 2004.

[8] R. Luce and H. Raiffa. Games and Decisions. J. Wiley, New York, 1957.

[9] E. Maskin. Decision-making under ignorance with implications for social choice. Theory and Decision, 11:319-337, 1979.

[10] W. Thomson. Cooperative models of bargaining. In R. Aumann and S. Hart, editors, Handbook of Game Theory with Economic Applications, pages 1237-1284. North Holland, 1994.

[11] A. Wald. Statistical Decision Functions. John Wiley, 1950. 